\title{
Maastricht Type 2 Donors: Unrealized Opportunities
}

\author{
J. D. Punch ${ }^{\mathrm{a}, *}$ and C. D. Anderson ${ }^{\mathrm{b}}$ \\ a Department of Surgery, University of Michigan, Ann \\ Arbor, MI \\ b Section of Abdominal Transplant Surgery, Washington \\ University in St. Louis, St. Louis, MO \\ *Corresponding author: Jeffrey D. Punch, \\ jpunch@umich.edu
}

\section{Received 27 June 2011, revised 23 September 2011 and accepted for publication 26 September 2011}

The 2006 report by the Institute of Medicine (IOM), titled "Organ Donation, Opportunities for Action" (1) estimated that 22000 of the 335000 individuals who die annually from cardiac arrest outside of a medical facility would be suitable for organ donation. Currently donation after cardiac death (DCD) in the United States usually occurs in Maastricht Type 3 donors that die after planned withdrawal of futile cardiorespiratory support in a hospital setting. This has also been termed "controlled" DCD because the timing of the donation event is planned. Maastricht Type 2 DCD, termed "uncontrolled" because the timing of the donation event is unplanned, occurs when an individual suffers cardiac arrest outside of a hospital, receives cardiopulmonary resuscitation en route to a medical facility, but the attempted resuscitation is unsuccessful and death is declared by circulatory criteria. The IOM report suggested that the expansion of Type 2 DCD in the United States should be an imperative.

The IOM also recommended that the term "uncontrolled DCD" be discouraged because it can be misunderstood to imply a lack of procedure or protocol. Rather the terms DCDD for "donation after circulatory determination of death" and DNDD for 'donation after neurological determination of death' were advocated because they make it clear that there are two acceptable methods of determining death-either no circulation, or no brain function. These terms have neither caught on in the organ donation nor in the Transplant community. The circulatory definition of death was recommended because "cardiac death" is a less precise term. Death of the cardiac tissue has not necessarily occurred at the moment that asystole occurs and restoration of the flow of oxygenated blood through coronary circulation may restore cardiac function. The most impressive demonstration of this is the fact that hearts have been used from donors that were said to have had "cardiac death".

There are two major hurdles to the development of Type 2 DCD within the United States; one is practical and the other is medical. The practical problem is the enormous amount of effort and coordinated engagement on the part of multiple parties that traditionally are not involved with organ donation that is necessary to develop such programs. In parts of Spain, the Transplant community has invested this effort and organs from Type 2 DCD are becoming a nontrivial portion of all organs available for transplantation there, particularly kidneys. The second barrier is the medical concern about warm ischemia. The concern over ischemia is particularly great for liver grafts because of the devastating complication of biliary cholangiopathy (2). To alleviate this concern, the Barcelona group has applied normothermic extracorporeal membrane oxygenation (ECMO) in the setting of Type 2 organ donation. It is notable that this protocol was developed in Spain, a country that already excels in organ donation effectiveness, as measured by donors per million. This is a testament both to the support of the Spanish populace for donation and for the dedication of the Spanish medical community.

In this issue, the Barcelona group report on 34 liver transplants performed over an 8 year period from Type 2 DCD (3). Their protocol involves the initiation of ECMO after cessation of cardiac massage. They observed 1-year graft and patient survivals of $71 \%$ and $80 \%$, respectively, results that are comparable to results of liver transplantation from Type 3 DCD but below that obtained with livers from brain dead donors. Importantly, only three patients (8\%) in this series developed ischemic cholangiopathy, a comfortingly low number.

Normothermic ECMO has also been used to support Type 3 DCD with excellent results (4) and offers logistical advantages over a rapid recovery technique. These advantages include a reduction in warm ischemia time due to the fact that oxygenated flow to the organs can be restored at the moment death is declared, rather than waiting until the abdomen has been opened and the aorta cannulated and clamped. This advantage is small if the withdrawal of support occurs in an operating room but greater if the withdrawal occurs elsewhere or if rapid aortic cannulation is not possible for technical reasons. The use of postmortem ECMO also allows withdrawal of support to occur in an intensive care unit setting without compromising organ quality. Although promising, it is not yet clear whether the 


\section{Punch and Anderson}

benefit of ECMO justifies the complexity and cost in the Type 3 DCD setting. However, in the Type 2 setting it is logical that ECMO will be necessary to achieve acceptable degrees of warm ischemia. The Barcelona group has demonstrated this elegantly with animal models (5).

Two elements of the Barcelona protocol raise potential ethical dilemmas and will doubtlessly require modification to be adopted in the United States. One is that the Barcelona protocol allows cannulation of the patient before family consent while cardiopulmonary resuscitation (CPR) is ongoing. Although this can be viewed as preserving the patient's option of donation, it was the IOM's stated view that consent should be obtained before invasive procedures are performed solely in the name of organ donation on patients that are still alive. This problem could be obviated however, if the donor has provided first-person consent for organ donation by signing up on a state registry, in that case permission of the family is not needed. It is also possible that family consent may be attainable before the end of attempted resuscitation.

The second element that is problematic about the Barcelona protocol is that cardiac massage and ventilation are continued after the declaration of death to avoid warm ischemia before the initiation of ECMO. Again, it can be argued that this is acceptable because it preserves the option of donation. However, many would argue that the resumption of circulation to the brain would mean that the patient is no longer officially dead if the IOM definition equating death to the absence of circulation to the brain is used, because circulation to the brain has resumed with CPR. ECMO too would generate the same concern for DCD if used without an aortic occlusion balloon to prevent circulation to the brain. Given that a 5 min period of asystole is allowed before CPR is resumed in the Barcelona protocol, it is very likely that whole brain function would be absent if it were possible to test for it after CPR resumes. Nevertheless, it is unpalatable to assume that the patient is brain dead when brain function has not been formally assessed.
A potential modification to the Barcelona protocol that would avoid this conundrum would be to begin cannulation for ECMO immediately upon declaration of death. This would avoid the resumption of CPR and, thus, avoid brain perfusion. However, it would be necessary to have the ECMO team ready and waiting very nearby and cannulation would have to be by cut down on the femoral vessels in the absence of blood flow. Alternatively, the patient could be 'redeclared' dead at the time that CPR ceases, something that is bound to be prohibitively confusing to medical teams and families.

The Barcelona group is to be congratulated for developing and demonstrating a successful strategy for the use of Type 2 donors. However, reproducing this level of success in the United States will require a large and coordinated effort from the medical community as a whole to satisfy the ethical and cultural concerns.

\section{Disclosure}

The authors of this manuscript have no conflicts of interest to disclose as described by the American Journal of Transplantation.

\section{References}

1. Organ donation. Opportunities for action. Washington, DC: The National Academies Press, 2006.

2. Jay $C L$, Lyuksemburg $V$, Ladner DP, et al. Ischemic cholangiopathy after controlled donation after cardiac death liver transplantation: A meta-analysis. Ann Surg 2011; 253: 259-264.

3. Fondevila C, Hessheimer AJ, Flores E, et al. Applicability and results of Maastricht type 2 donation after cardiac death liver transplantation. Am J Transplant 2012; 12:162-170.

4. Magliocca JF, Magee JC, Rowe SA, et al. Extracorporeal support for organ donation after cardiac death effectively expands the donor pool. J Trauma 2005; 58: 1095-101; discussion 101-102.

5. Net M, Valero R, Almenara R, et al. The effect of normothermic recirculation is mediated by ischemic preconditioning in NHBD liver transplantation. Am J Transplant 2005; 5: 2385-2392. 